\title{
Hexagonal Uniformly Redundant Arrays (HURAs) for Scintillator Based Coded Aperture Neutron Imaging
}

\author{
Kelum A.A. Gamage, Member, IEEE, Qi Zhou, S.C. Hayes
}

\begin{abstract}
A series of Monte Carlo simulations have been conducted, making use of the EJ-426 neutron scintillator detector, to investigate the potential of using hexagonal uniformly redundant arrays (HURAs) for scintillator based coded aperture neutron imaging. This type of scintillator material has a low sensitivity to gamma rays, therefore, is of particular use in a system with a source that emits both neutrons and gamma rays. The simulations used an AmBe source, neutron images have been produced using different coded-aperture materials (boron10, cadmium-113 and gadolinium-157) and location error has also been estimated. In each case the neutron image clearly shows the location of the source with a relatively small location error. Neutron images with high resolution can be easily used to identify and locate nuclear materials precisely in nuclear security and nuclear decommissioning applications.
\end{abstract}

Index Terms-Hexagonal uniformly redundant arrays, codedaperture neutron imaging, Monte-Carlo simulations, EJ-426 detector

\section{INTRODUCTION}

$\mathbf{S}$ CINTILLATOR based coded-aperture imaging is currently more popular using gamma rays than neutrons. Previous work conducted with gamma rays on detecting a source location have proven to be successful [1] and even some implementations of MURA (Modified Uniformly Redundant Array) patterns with a ${ }^{3} \mathrm{He}$ proportional counter detector have produced accurate results [2], however due to previous problems experienced with gamma-ray sensitivity, work with scintillators in accurately locating a neutron source has been largely unexplored.

Generally scintillator based neutron detectors, fast organic liquid scintillator detectors, are sensitive to both neutrons as well as gamma rays. This limits the uses of these scintillator based detectors in coded-aperture applications. However, recent advances in digital pulse shape discrimination methods enable the use of such scintillators in mixed-radiation imaging [3]. Pulse shape analysis determines whether the event was caused by a neutron or gamma ray based on the decay characteristics of the recorded event. This also encourages the use of scintillators (such as EJ-426 neutron scintillator), with high sensitivity to neutrons (compared to gamma rays), in high resolution coded-aperture neutron imaging applications.

Manuscript received April 6, 2015. This work was supported by the Department of Engineering, Lancaster University, UK.

K.A.A. Gamage and Q. Zhou are with the Department of Engineering, Lancaster University, Lancashire, LA1 4YW, UK (telephone: 0044-1524593873, e-mail: k.gamage@lancaster.ac.uk).
One of the advantages of the use of coded-aperture's is that if made from a shielding material they can successfully moderate or stop the respective source particle, which in this case is of course neutrons. This respectively eliminates the need for a collimator to protect the detector from scattered or background radioactivity. However, one of the largest problems with neutron sensitive scintillator detectors is the vulnerability they have to detecting gamma rays, as a likely side product of neutron decay is gamma rays it causes a worrying problem, as stray counts are recorded via an unwanted source particle. Recent developments in pulse shape analysis however reduce if not eliminate this problem by allowing the user to identify which particle has caused an interaction. This therefore encourages the use of scintillators that are sensitive to neutrons compared to gamma rays. Such a scintillator would be the EJ-426 which consists of ${ }^{6} \mathrm{LiF}: \mathrm{ZnS}$ [4].

The three most common geometrical configurations used for the coded-aperture are linear, square and hexagonal, where complexity of the coded-aperture as well as the accuracy of the imaging system increase in the same order (i.e. square is better compared to linear and hexagonal is better compared to square) [5]- [7]. For example, it enables more adaptability for differing situations by providing more options when imaging complex shapes. The linear coded-apertures are symmetrical about their centres providing a mirror like symmetry. The square coded-aperture masks are invariant about 180 and the hexagonal coded-aperture masks are symmetrical about a 60 rotation through their centres. It was believed that these properties were key in terms of future developments, such as the construction of future patterns and the development of new, faster decoding algorithms [7]. The development of the MURA brought many more sizes of apertures into the field. This led to a surpassing of the constraints which limited the URA, enabling users a much wider selection of pattern, with a higher chance of being able to select a pattern that matches the particular detector constraints.

In this paper we have modelled and simulated a neutron imaging system using a Monte Carlo simulation package (MCNPX) and with a Hexagonal Uniformly Redundant Array (HURA) type of coded-aperture. The mathematical structure and property of the hexagonal coded-aperture is discussed in the coded-aperture design section where details of the modelling and simulation is included in the Monte Carlo modelling and simulation section. The neutron images produced with respect to boron-10, cadmium-113 and gadolinium-157 codedapertures are included in the result section. 


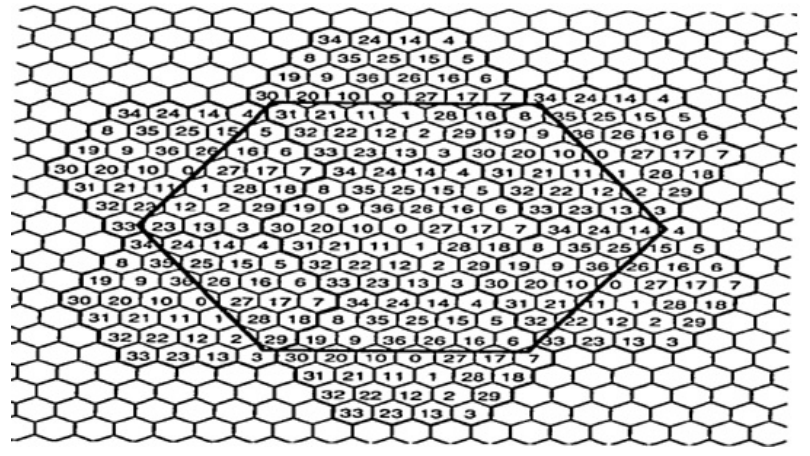

(a)

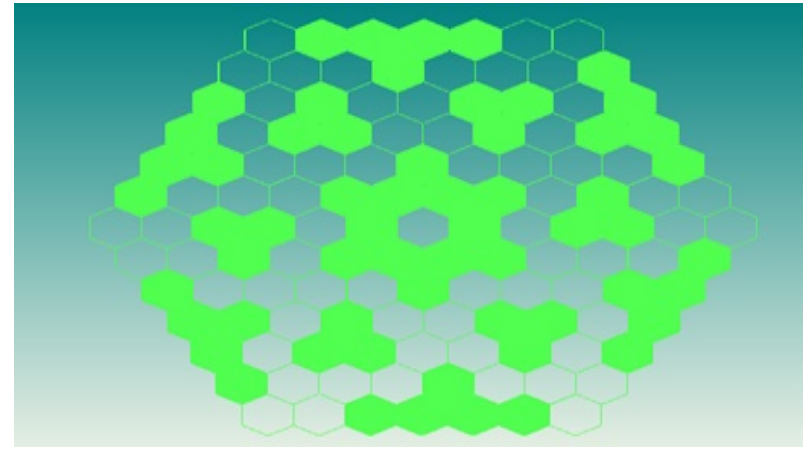

(b)

Fig. 1. (a) Infinite hexagonal grid with a hexagonal lattice array of 37 elements; (b) relative status of each cell in the hexagonal lattice array, where white area indicates the cell is open and black area indicates the cell is closed.

\section{Coded Aperture Design}

The modified Uniformly Redundant Array (MURA) pattern, decoding pattern and general layout of simulating system are the key designing components of the scintillator based codedaperture neutron imaging system. In order to develop the MURA pattern it is necessary to evaluate several equations, which dictate the open and closed sections of a typical two dimensional coded-aperture binary array. It is therefore important that the design of the MURA pattern is of sufficient quality to enable the encoding of the source data before interaction in the detector. The MURA also moderates the neutrons of the system.

In this work, a hexagonal coded-aperture has been considered to improve the accuracy of the proposed neutron imaging system. The design of the hexagonal coded-aperture completed with use of the Skew-Hadamard URA construction concept as well as considering the symmetrical nature (60 rotation through their centres) of the hexagonal URA [8]. Figure 1(a) shows infinite hexagonal grid with a hexagonal lattice of 37 element array. The hexagonal pattern is similar with respect to 60 degree rotation around its central element. The central element is always 0 in the hexagonal modified uniformly redundant array, where all cell numbers in a diagonal line increase from the top to the bottom. These cell numbers are cyclic, for example when the cell number reaches the maximum number (36 for the case of 37 element array), the next cell number set to 0 . The state of each cell in the hexagonal lattice in terms of whether the cell is open or closed can be represented as shown in Fig. 1(b) based on linear modified uniform redundant encoding array [7].

As the MURA has the function of encoding the source data for detection, it is necessary that once the data is recorded by the detector a decoding technique is performed. This should allow recovery of the original source information. With MURA patterns the development of the decoding array is made relatively simple. For any given size of MURA, there is a individual decoding pattern that can be found by applying a series of equations to the respective MURA design [7]. The corresponding decoding array for the hexagonal lattice is also similar to the case of linear modified uniform redundant decoding array [7], where it can be represented as shown in
Fig. 2, for example " + represented " +1 in the decoding array and "- represented " -1 in the decoding array.

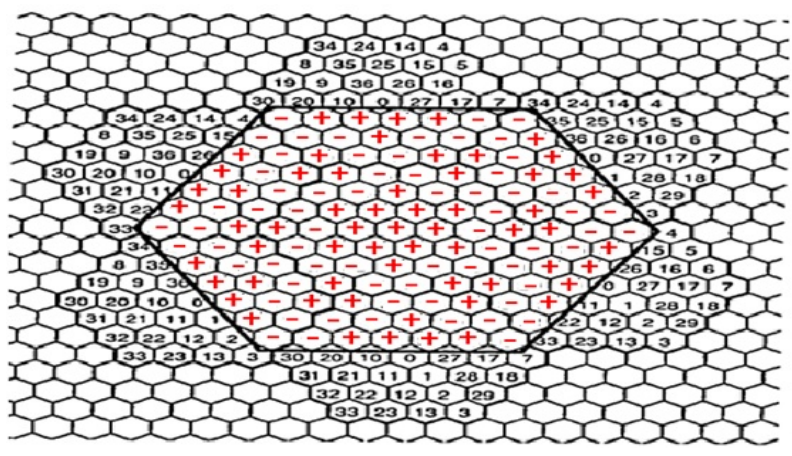

Fig. 2. The corresponding decoding array for the hexagonal lattice

\section{Monte Carlo Modelling and Simulation}

A neutron imaging system, that utilises a hexagonal geometry modified uniformly redundant array for the coded-aperture, has been modelled and tested using MCNPX. The design of the more complex geometries was done with MCAM software, a program that allows conversion between CAD drawings and MCNPX code. As shown in Fig. 3, a neutron source, a codedaperture and a scintillator were simulated using MCNPX.

In this study the coded-aperture was modelled for three different materials namely: boron-10, cadmium-113 and gadolinium-157, and each case the simulations have been conducted making use of EJ-426 neutron scintillator detector. In total there were 127 cells in the 37 element hexagonal lattice; 60 cells were open cells and 67 were closed cells (i.e. $47.24 \%$ aperture element is transparent to the incident radiation), where dimension of each side of the hexagonal cell was $5 \mathrm{~mm}$. It is also important that the scintillator detector is position sensitive, in that during the convolution process the point at which each neutron has interacted is needed to obtain the original source information. Therefore, the scintillator was also modelled with 127 cells with equal dimensions, with a thickness of $25 \mathrm{~mm}$ separated from the coded-aperture by a distance of $5 \mathrm{~cm}$. The source was modelled as an AmBe point 
source, where it placed $10 \mathrm{~cm}$ away from the center point of the coded aperture as shown in Fig. 3 (where each MCNPX simulation run comprised 10 million particle histories using a computer with Windows 7 edition and with 4 GB RAM). The individual cells of the MURA all aligned perfectly (with the corresponding cells in the scintillator) and then perform the simulations setting a tally to score the number of neutrons interacting in each particular cell. This is then printed as a result in the output file.

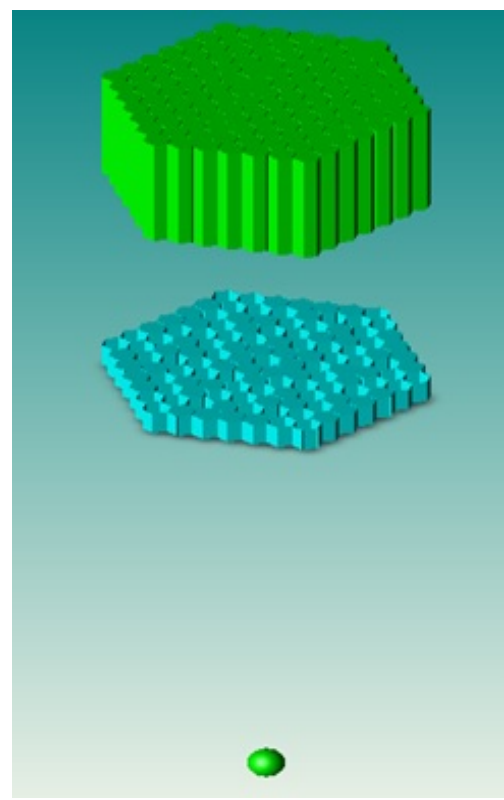

Fig. 3. Illustration of the simulation model with the coded-aperture, scintillator and the neutron source.

\section{RESUlts AND Discussion}

MCNPX simulated results can be convoluted with an inverse pattern of the MURA array giving the precise location of the point source [7]. The resulting radiation images produced in this research are shown in Fig. 4. It is clear that the system can locate the source in each case, where red spot on each image corresponding to the reconstructed location of the source. The error of the reconstructed location of the source with respect to the location of the original source is calculated and tabulated in Table I.

TABLE I

CALCULATED DISTANCE ERROR OF THE RECONSTRUCTED LOCATION OF THE SOURCE, WHERE ORIGINAL LOCATION OF THE SOURCE IS $(0,0)$.

\begin{tabular}{ccc}
\hline $\begin{array}{c}\text { Coded-aperture } \\
\text { material }\end{array}$ & $\begin{array}{c}\text { Reconstructed location } \\
(\mathbf{Y ~ c m}, \mathbf{Z} \text { cm })\end{array}$ & $\begin{array}{c}\text { Distance error } \\
(\mathbf{d} \text { cm })\end{array}$ \\
\hline Boron-10 & $(0.1,1.6)$ & 1.603 \\
Cadmium-113 & $(0.2,1.5)$ & 1.513 \\
Gadolinium-157 & $(0.8,0.0)$ & 0.800 \\
\hline
\end{tabular}

According to the estimated distance errors, gadolinium-157 coded aperture offers relatively high accuracy compared to boron-10, and cadmium-113 coded-apertures. As expected,

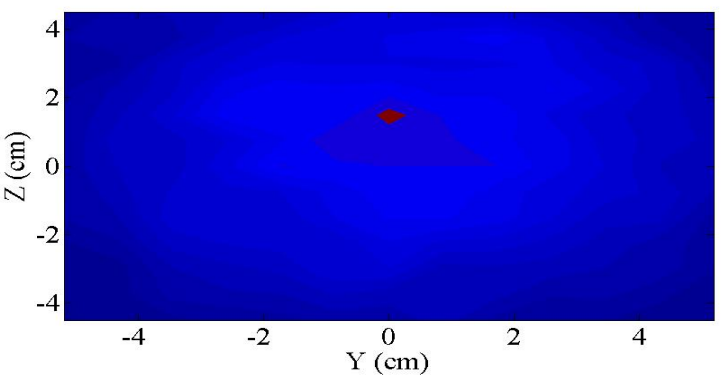

(a) aperture type: boron-10

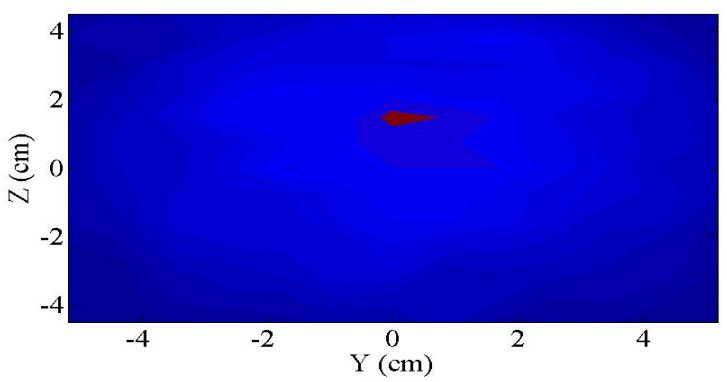

(b) aperture type: cadmium-113

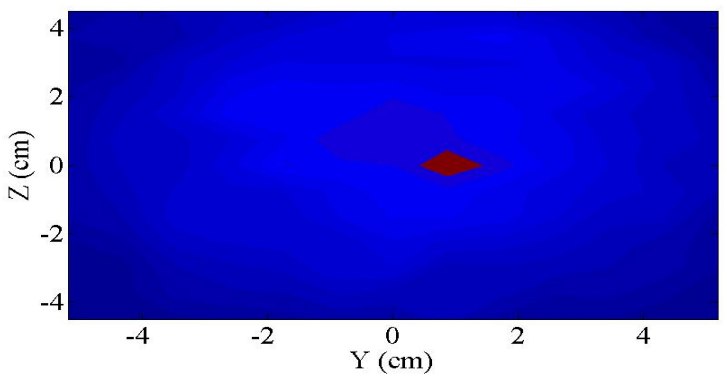

(c) aperture type: gadolinium-157

Fig. 4. The radiation images produced from convoluted MCNPX simulations, where the dark read spot on each image represents the reconstructed location of the source. In each simulation, source original location was at $(0,0)$

due to its particularly high neutron capture cross-section (259000 barns), the gadolinium coded-aperture based system operates with the lowest error on the reconstructed source location with regards to the actual location of the source. Boron and cadmium coded-aperture based systems are also produced relatively accurate results. However, errors are similar irrespective of the high capture cross section of the cadmium (64000 barns) with respect to boron (3835 barns).

Scintillator based HURA coded-aperture system has been able to reconstruct a source location via the convolution of recorded data (in the form of neutron count) with a relatively high accuracy. The size and hexagonal shape of the MURA pattern has also proved sufficient to encode the neutrons emitted from the AmBe source. The potential applications for this type of system could be numerous, for example to detect and locate neutron sources in the fields of nuclear decommissioning and nuclear security. 


\section{ACKNOWLEDGMENT}

The authors would like to acknowledge the financial support of Engineering Department, Lancaster University, UK. The authors would also like to acknowledge the the use of the package MCAM.

\section{REFERENCES}

[1] D.N. Anderson, D.C. Stromswold, S.C. Wunschel, A.J. Peurrung and R.R. Hansen, "Detection and location of gamma-Ray sources with a modulating coded mask," Technometrics, vol. 48, pp. 252-261, 2006.

[2] P.E. Vanier, "Improvements in coded aperture thermal neutron imaging," in SPIE Conf. Proc., vol. 5199, 2004.

[3] K.A.A. Gamage, M.J. Joyce and N.P. Hawkes, "A comparison of four different digital algorithms for pulse-shape discrimination in fast scintillators," Nucl. Instr. and Meth. A, vol. 642, pp. 78-83, 2011.

[4] EJ-426 Data Sheet, Eljen Technology, Sweetwater, Texas, 2013.

[5] E.E. Fenimore and T.M. Cannon, "Coded aperture imaging with uniformly redundant arrays," App. Opt., vol. 17, pp. 337-347, 1978.

[6] T.M. Cannon and E.E. Fenimore, "Coded aperture imaging with uniformly redundant arrays," App. Opt., vol. 17, pp. 337-347, 1978.

[7] S.R. Gottesman and E.E. Fenimore, "New family of binary arrays for coded aperture imaging," App. Opt., vol. 28, pp. 4344-4352, 1989.

[8] M.H. Finger and T.A. Prince, "Hexagonal uniformly redundant arrays for coded-aperture imaging," Proc. of 19th Intern. Cosmic Ray Conf., vol. 3, pp. 295-298, 1985. 